\title{
Navigation of robotic platform for gait disorders monitoring
}

\author{
J. Crha, O. Ťupa, J. Mareš, A. Procházka \\ Department of Computing and Control Engineering \\ University of chemistry and technology Prague \\ Prague, Czech Republic \\ jan.crha@vscht.cz
}

\begin{abstract}
This paper is focused on control of a six-wheeled robotic platform used for gait pattern recording. Navigation of the platform has to be very precise to keep the path of the platform as narrow as possible and to maintain the distance between the subject and the platform to capture the gait pattern in the most natural way. A key component of the whole robotic platform is an MS Kinect $v 2$ device which tracks the patients' gait. Kinect also provides the information about the distance between the robot and the patient to a microcontroller which is responsible for the wheels' control. To obtain straight trajectory, there is a camera pointing forwards on the front of the robot and a set of two beacons at the end of the operation area to be tracked by the camera. All data obtained from the camera, Kinect and encoders (attached to each wheel) are used during an offline reconstruction of the patients' gait. To process and save the data form Kinect, camera and other controllers in real-time a special multithread application was developed.
\end{abstract}

Keywords-component; robotic platform; gait tracking; Kinect v2

\section{INTRODUCTION}

Modern medicine willingly benefits from the development of computer vision systems. It is mainly because these offer all sorts of easy and effective noninvasive diagnostic methods, such as observing and analyzing gait patterns. The Alzheimer's or Parkinson's diseases manifest, among other things, by disarraying the stride - step length, coordination of limbs, etc. Computer vision allows us to detect certain symptoms in early stages of these diseases, thus providing in time diagnostics.

Many papers deal with the Parkinson's disease and its influences on the stride and body holding in this period. There are several ways how to observe the movements or the gait of patients, these systems are mentioned in many papers [1,2,3]. From the neurologist point of view, the gait on a treadmill or with special equipment attached directly to the body of the person is different from the natural one. For example, the difference walking on a treadmill makes in the inner ear movement data processing. The inner ear is a complex sensor of movements and it takes care of the stability. Walking on a treadmill causes that the inner ear sends information that the body does not move, but the legs do. This situation is confusing for the brain.
This paper deals with one of the possible ways how to eliminate these disadvantages. The patients' gait is tracked from a mobile robotic platform. This robotic platform was developed to eliminate main issues and it is designed to capture the movement of the whole body during natural walking. The Kinect v2 sensor is used for the subject tracking. It is able to calculate the $3 \mathrm{D}$ position of the 25 major joints.

This approach seems to be promising from the neurologist point of view, but it causes some problems with the gait reconstruction:

- The absolute position of both observed the subject and the sensors have to be calculated.

- The path of the robotic platform has to be as narrow as possible, for an easier gait reconstruction

- The platform must avoid of any shakes, for higher video and reconstruction quality

Similar projects of body tracking systems were already created like a Kinect navigated robotic platform used in [1] to follow elderly people. The authors Stone E. and Skubic M. proposed an application of the Kinect sensor for an in-home gait measurement to prevent people with Parkinson's disease from falling [2]. Kinect is also used for tracking speed skaters [3] to collect data for their trainers. The authors use a standard RC car as a carrier for their equipment, including the Kinect sensor. All aforementioned authors use infra-red and/or depth data provided by Kinect for their tracking algorithms. Another way to track people is in $[4,5]$ where a stereoscopic camera is used and in [6] even combined with a Laser Range Finder (LRF). The authors use feature detection to extract individuals from the captured images. After that, the position of the selected region, the colour and the texture of their clothes are used for improving recognition in subsequent images. LRF is also used as an obstacle avoiding system and its data are fused with the camera system data to obtain even more precise results.

\section{MOTIVATION}

Gait analysis involves measurement, extraction of well-describing features and interpretation of the results leading to a conclusion about the health of the subject. The process of measurement is highlighted in this paper. There are several techniques how to 
observe the gait pattern. There are two main categories of the features that have to be observed during gait tracking.

Temporal and spatial measurement provides the features such as walking speed or stride length. This process is usually carried out by a stopwatch and marks on the ground, walking on a pressure mat, laser sensors placed few centimetres above the ground or inertial sensors (gyroscopes and accelerometers).

Measurement of Kinematics could be performed by following methods:

- Chronophotography is using strobe lighting at a known frequency and capturing the images

- Cine film or video recordings from a single or multiple cameras to measure joint angles and velocities. This method allows an analysis in three dimensions.

- Passive marker systems consist of reflective makers and multiple cameras (up to 12) sensitive to the reflection of used materials (usually red, infrared or near infrared).

- Active marker systems are based on a similar philosophy as the passive markers with the exception that the markers are triggered by the incoming signal.

- Inertial systems do not need any camera and the movement is captured by a set of sensors. Each sensor is a combination of a gyroscope and an accelerometer. The gait tracking is inferred according to a biomechanical model and a fusion of the information from all sensors.

All these methods need some special equipment of the laboratory, wearable sensors limiting the movement or sensors dedicated to one purpose. The disadvantage is the limitation of the Kinect's field of view, enabling reliable tracking of people only in the distance between $0.5 \mathrm{~m}$ and $4.5 \mathrm{~m}$. Therefore the combination of the cost-effective Kinect sensor and a mobile robotic platform is opening a new horizon to track the gait pattern.

In the previous studies $[5,6]$ the Kinect device was placed stationary on a table to track people in its field of view. The MS Kinect that was used for data acquisition was installed approximately $60 \mathrm{~cm}$ above the floor. Each individual repeated a straight walk of approximately $4 \mathrm{~m}$ (five steps) back and forth 5 times. The experimental part of this study was devoted to the analysis of the gait of the following two sets of individuals: 18 patients with Parkinson's disease and 18 healthy age-matched individuals. From the acquired records 5 features were extracted - walking speed, stride length, centre of mass deviation in the horizontal and vertical projection and limb coordination. The results were obtained by a combination of these characteristics by a neural network and the accuracy of decision of this system was up to $91.7 \%$.

\section{EQUIPMENT OF PLATFORM}

The platform was developed as a six-wheeled off-road vehicle that participated in autonomous robot contests. The vehicle was equipped with ultrasonic sensors which were used as an anti collision system. Other sensors were a GPS, a magnetic sensor and encoders on each wheel. These sensors were used for navigation and movement control.

The platform was upgraded in 2015 using the Kinect v2 sensor and a camera. RaspberryPi was replaced by a powerful notebook, which was able to handle Kinect v2's requirements and new code for whole platform was developed, to implement new hardware functions and to change the focus from an outdoor vehicle, to a medically oriented platform used for patients' gait tracking. To correspond with the requirements of all equipment, new axes were created and even the wheels were switched to a new type.

Nowadays the platform is equipped with narrow wheels of $145 \mathrm{~mm}$ in diameter that are connected through gearboxes to motors. Gearboxes reduce speed of motors to $140 \mathrm{rpm}$ and increase stall torque to $0.8 \mathrm{Nm}$ with gear ratio about 1:75. Each motor is equipped with its own motor driver and encoder. The motor driver is designed to provide a maximum of $5 \mathrm{~A}$, using a PWM to control the power. Two mbed LPC1768 boards control the motors; one of them is only a coprocessor of signals from all encoders and the second one computes the desired speed and PWM duty cycle for each wheel, based on information from the encoders and also from the notebook. The latter provides information about the distance to the patient and the direction of the platform. Platform overview can be seen in Fig. 1.

The mbed is powered by an NXP chip LPC1768, which is an ARM Cortex-M3 processor, running on $96 \mathrm{MHz}$ and with a $512 \mathrm{kB} \mathrm{FLASH}$ and a $64 \mathrm{kB}$ RAM. It has 30 GPIO pins, which is enough for all safety switches, ultrasonic range finders and also for communicating with six motor driver boards via an SPI, but quadrature encoders must be connected to the "slave" board, because of another 12 lines. Each encoder provides 48 pulses per revolution of the motor shaft, which means that at full speed of motors, all encoders generate over 50 interrupts per millisecond. In this arrangement, master board is able to run control loop every $20 \mathrm{~ms}$, but with encoders connected directly to master board, maximal time for computing

Figure 1. Actual photography of the platform.

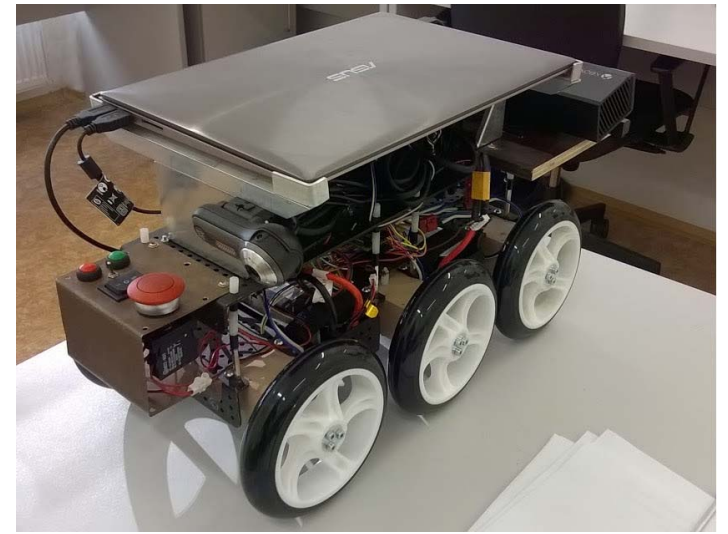


control loop depends on speed of platform and its maximal value (at full speed) is about $140 \mathrm{~ms}$ furthermore problems with serial communication appears.

Platform is also equipped with two different batteries, where one is connected to a separate DC-DC converter dedicated to the MS Kinect v2 sensor, and a second one connected to a custom made power distribution PCB. Four more DC-DC converters are placed on the $\mathrm{PCB}$, and also several high current terminals for powering the motor drivers. There are five different voltages available on the platform:

- $\quad 3.3 \mathrm{~V}(2 \times 300 \mathrm{~mA})$ regulated, for motor driver boards and other electronic chips.

- $\quad 5 \mathrm{~V}(2 \mathrm{x} 300 \mathrm{~mA}, 1 \times 2 \mathrm{~A})$ regulated, for mbed boards, ultrasonic sensors and other possible accessories like a Bluetooth dongle or a RaspberryPi.

- $12 \mathrm{~V}(1 \mathrm{x} 4 \mathrm{~A})$ regulated, for the Kinect v2 sensor

- $\quad 7.4 \mathrm{~V}$ unregulated, directly from the first battery, used for powering the motors and DC-DC convertors for 5 and $3.3 \mathrm{~V}$.

- $\quad 18.5 \mathrm{~V}$ unregulated, directly from the second battery. This source is used only for the 12 V DC-DC convertor for Kinect.

The platform carries a Kinect v2 sensor pointing backwards, which is crucial for the gait reconstruction. The Kinect weighs $1.8 \mathrm{~kg}$, and another $1.5 \mathrm{~kg}$ of weight are added by a notebook. To handle additional weight, the former independently suspended half-axes were replaced by custom made hard axes, to prevent swinging of the sensors when accelerating or decelerating.

A camera is pointing forwards and its images are used to detect two navigational lights which are placed on the opposite side of the corridor where gait monitoring takes place. Data from the camera and from Kinect are processed and saved in an Asus Zenbook, which is equipped with an Intel core i7 processor, $8 \mathrm{~GB}$ of RAM, USB 3.0 and a 256 GB SSD. Only this configuration was acceptable to suit the requirements of Kinect v2 and to be able to process and save smoothly all the required information gathered from the sensors and necessary for the gait reconstruction. Data transmission over the entire platform can be found on Fig. 2.

Figure 2. Data transmission in robotic platform

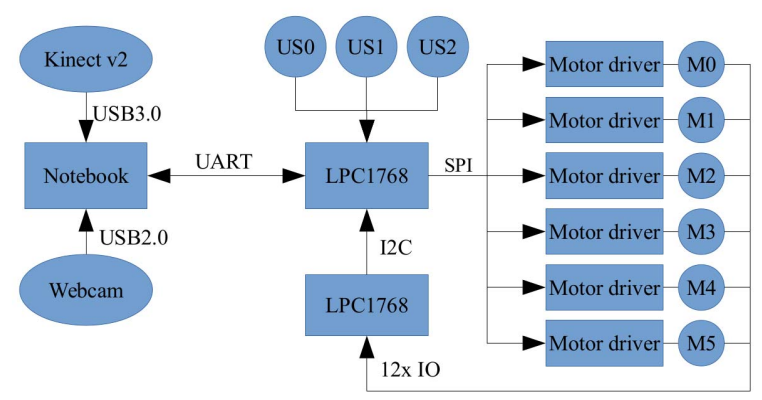

In Fig. 2 every module is blue, modules that provide some information are round and in rectangles data processing is done. Standard buses have labels and non labelled vertexes are custom connections. Type of transmitted information will be discussed in next chapter.

\section{CONTROL OF PlatForm}

The "brain" of the platform is the Zenbook, where a custom multithread application is running. This application is written in $\mathrm{C \#}$ and it gathers infra-red and depth images from Kinect, RGB images from the camera and data from all encoders provided by the mbed boards. Kinect, camera and also mbed connected to Zenbook are all asynchronous sources, so there are three separate threads that take care of each data source and another three threads for saving processed data. First of logger threads is a runtime logger, where all the events and messages are logged, e.g. time when the Kinect data were saved, when a message from mbed was received, what was found on the camera image and what was sent to the mbed as new navigational data. The second logger saves binary images from Kinect with attached time stamps and distance stamps. The distance stamps are raw distances in $\mathrm{mm}$ travelled by all platform wheels. The third logger saves camera images in full resolution. Thanks to this distribution, the entire application runs smoothly and everything is done in the shortest time possible. The application also contains a well-arranged graphical user interface, where the state of all the connected sensors and boards is shown. It also allows showing previews from Kinect, the camera and the communication with the mbed master board.

Navigational data are composed from both Kinect images and camera images. Camera images are used to detect the navigational lights and then compute the deviation of the platform's direction. The navigational lights are two super bright LEDs that are placed on the opposite side of the corridor where the measurement takes place. Each image is resized, smoothed with blur filter and then threshold over each layer is done. Only pixels which are in range in all layers together are used for contours detection and then convex hull and enclosing circles are computed for each object. Both contours and circles are filtered to obtain only two objects, which position in image is computed and position of corridor centre is derived. Kinect images are processed to obtain the distance between the patient and the platform. The patient is wearing IR light reflective markers on their arms and legs for a robust detection of position. Both the direction deviation and the distance from the patient are sent to the mbed that computes new coefficients for the speeds of all wheels.

The total of eight control loops is placed in the master mbed. When mbed receives an update with the distance from the human and direction deviation, first it computes difference of platform and human speed. This value is than added to current speed set point and if distance is outside required range, additional proportional value is added or subtracts from speed set point. Direction deviation is then transformed to a unit less coefficient which is applied 
to the speeds of one side of the platform and its inverse value to the other side.

All six speed set points are set and then they are forwarded to six proportional controllers which return outputs in a deviation form. Each proportional controller then computes a new PWM duty cycle for each motor driver based on the actual speed of the connected wheel, to correspond with the actual speed set point. After the regulators are tuned, mean error of the wheel position is about $1 \mathrm{~mm}$ and it rarely goes over $3 \mathrm{~mm}$.

\section{GAIT RECONSTRUCTION}

The following step is post-processing of each record to obtain gait pattern. Gait reconstruction is performed in MATLAB environment and additional robust scripts are used to merge data computed during measurement with information gathered from entire dataset.

Detection of limb position is performed on infrared images. Thanks to infrared markers, limbs are detected precisely, and due to same resolution and point of view of both the infrared camera and the depth sensor, positions of limbs in each pair of images are the same. Fig. 3 documents this process.

Absolute position of limbs is derived from the relative position and actual distance driven and the angle of the platform. Angle between the subject and the platform is computed from RGB images by

Figure 3. Detection of relative limb position using infrared and depth data. The absolute position is a combination of the distance driven gathered from the encoders and the relative position received from Kinect.
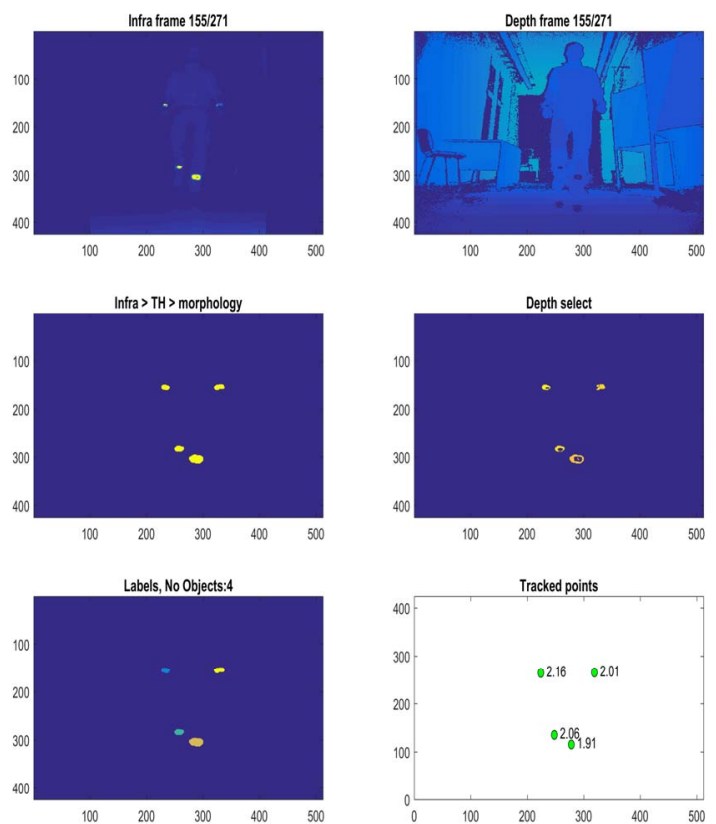

cross-covariance used on parts with the two lighthouses. The gait features are extracted from the absolute positions of the limbs Proposed features are gait velocity, stride length, left and right leg step length and limbs co-movement. Further information about gait reconstruction with static sensor was published in our earlier papers $[7,8]$.

\section{CONCLUSION}

Contemporary result of this project is the hardware solution of the robotic platform. This platform can perform autonomous measurements that are used for gait analysis. The platform is equipped with sensors that provide information about its environment and it can alter its behaviour in different scenarios. Also a database of measurements is being created from testing runs. This database is now being processed and first interim results are obtained.

The platform development is almost finished and the project is now in its last testing phase in the university background. Next it will be moved to the neurology department of the University Hospital in Hradec Králové. We are trying to automate the control of the platform and to create a user-friendly application for recording, which will be handled only by the staff of the hospital.

Financial support from specific university research (MSMT No 20-SVV/2016)

\section{REFERENCES}

[1] Machida E., Cao M., Murao T. and Hashimoto H., Human Motion Tracking of Mobile Robot with Kinect 3D Sensor, SICE Annual Conference, p.2207-2211, 2012

[2] Stone E. E. and Skubic M., Unobtrusive, Continuous, InHome Gait Measurement Using the Microsoft Kinect, IEEE Transactions on Biomedical Engineering, 60, 2925-2932, 2013

[3] Boyd, J.E., Godbout, A., Thornton, C., In Situ Motion Capture of Speed Skating: Escaping the Treadmill, Computer and Robot Vision, p.460-467, May 2012. ISBN: 978-1-46731271-4

[4] J. Satake and J. Miura, "Robust stereo-based person detection and tracking for a person following robot," in ICRA Workshop on People Detection and Tracking, Kobe, Japan, May 2009

[5] Z. Chen and S. T. Birchfield, "Person following with a mobile robot using binocular feature-based tracking," in International Conference on Intelligent Robots and Systems, San Diego, CA, October 2007, pp. 815-820.

[6] T. Sonoura, T. Yoshimi, M. Nishiyama, H. Nakamoto, S. Tokura, and N. Matsuhira, "Person following robot with vision-based and sensor fusion tracking algorithm," in Computer Vision, X. Zhihui, Ed. Vienna, Austria: InTech, 2008, ch. 27, pp. 519-538.

[7] A. Procházka, O. Vyšata, M.Vališ, O.Ťupa, Schatz, and V. Mařík. Use of the Image and Depth Sensors of the Microsoft Kinect for the Detection of Gait Disorders. Springer: Neural Computing and Application, 2014

[8] O. Ťupa. Multi-Dimensional Data modelling and Analysis Using MS Kinect. Diploma thesis, VŠCHT Praha 2014 